epilepticus in children; it is investigative and nonapproved for use in neonates. Prospective studies may be justified.

In an Editorial, Sankar R and Painter MJ (Neurology 2005;64:776-777) applaud the study of midazolam and the promising results. However, they raise concerns about potential neurotoxicity of GABA agonists in the immature brain. AMPA antagonists, such as topiramate, might be a safer class of drug to promote in IV form for trial in neonates.

\title{
BENIGN FAMILIAL INFANTILE SEIZURES
}

The history, classification, clinical and EEG features, genetics, differential diagnosis, and outcome of "benign familial infantile seizures" (BFIS) are reviewed from the Neurology Department, Bambino Gesu Children Hospital, Rome, Italy. First reported by Fukuyama (1963) as partial seizures occurring in the first 2 years, idiopathic, and with a benign outcome, the syndrome was later described as benign partial epilepsy of infanicy with complex partial seizures (BPE and CPS) and BPE with secondarily generalized seizures in infancy (Watanabe et al. 1987, 1990, and 1993). The term "benign infantile familial convulsions" was proposed by the present author (Vigevano F et al. 1992), cases showing an autosomal dominant inheritance. Finally, in the ILAE classification (2001) the term "benign familial infantile seizures" was preferred, and two forms were listed, familial and nonfamilial. The inheritance is heterogeneous, with chromosome markers on chromosomes 19, 16 (BIFC and paroxysmal choreoathetosis), and on chromosome 2. Two families were also described with onset at the $2^{\text {nd }}$ month, with mutations in the sodium-channel gene $\mathrm{SCN} 2 \mathrm{~A}$, and two with neonatal onset had genetic mutations associated with potassium channels. Some cases have been associated with diarrhea and rotavirus.

Clinical characteristics are as follows: Family history of similar seizures; normal early development; onset 3-10 months; normal neurologic exam; seizures in clusters; partial (occipito-parietal) seizures; normal interictal EEG; benign course; and normal developmental outcome. In the absence of a family history of BFIS, early diagnosis may be difficult and only by exclusion of possible etiological factors or by identification of the genetic marker. Sporadic cases may carry the same genetic marker as familial ones, with less expressivity. (Vigevano F. Benign familial infantile seizures. Brain Dev April 2005;27:172-177). (Respond: F Vigevano; E-mail: vigevano@opbg.net).

COMMENT. The syndrome of BFIS has characteristic clinical features and benign outcome but variable genetic mutations associated with a channelopathy. The decision to treat depends on the severity and frequency of seizures and the family history. The author cites untreated familial cases having isolated or brief clusters up to 1 year of age.

\section{PROGNOSIS OF BENIGN MYOCLONIC EPILEPSY OF INFANCY}

Neuropsychological, cognitive, and behavioral outcome was studied in a long-term follow-up of 7 patients with benign myoclonic epilepsy in infancy (BMEI) at Universita di Palermo, Italy. Mean age at onset of myoclonic seizures (MS) was 15 months (range, 7-35 months). Febrile convulsions had occurred before the onset of MS in 3 infants. Myoclonic jerks involved mainly the upper limbs, with nodding and upward gaze deviation in some, and flexing of the body and lower limbs. Ictal EEG recordings showed generalized spike-wave 
and/or polyspike-wave synchronous with myoclonus; interictal EEG was normal. Seizures were controlled by valproate, and medication was discontinued after a seizure-free period of 3 years. The average duration of follow-up was 6 years 9 months (range, 4 yrs 9 mos- 9 yrs 2 mos). At the end of follow-up, 2 children with early onset of seizures ( 7 months) had mild or moderate mental retardation, deficits in fine motor coordination, attention deficit disorder, and language impairment. Three children had a borderline IQ and 1 a low average IQ, with learning disorders and attention deficits. Only one patient had a normal IQ. All children were oppositional, irritable, aggressive, and hyperactive. The mean Full Scale IQ of the group was 74, 5 had ADD, and 4 had learning disorders. Early onset of seizures is a major risk factor for poor cognitive and behavioral outcome. A delay in starting treatment is an additional risk factor. (Mangano S, Fontana A, Cusumano L. Benign myoclonic epilepsy in infancy: neuropsychological and behavioural outcome. Brain Dev April 2005;27:218-223). (Respond: E-mail: manganos@katamail.com).

COMMENT. BMEI, as described by Dravet and Bureau (1981), is characterized by myoclonic seizures in previously healthy infants, 4-36 months of age, and responsive to antiepileptic medication. The unfavorable outcome reported in the above study compared to previous series was linked to early onset of seizures and delay in starting treatment. Alternatively, a pre-existing mild encephalopathy may have been responsible. The authors conclude that BMEI interferes with the growth of developing neurons, resulting in long-term neuropsychological impairment.

\section{BRAIN NEOPLASMS}

\section{POSTOPERATIVE CHEMOTHERAPY FOR MEDULLOBLASTOMA}

The survival rate and cognitive function of 43 children, age $<3$ years, with medulloblastoma treated with intensive postoperative chemotherapy alone, without radiotherapy, were determined at the University of Wurzburg and other centers in Germany Chemotherapy consisted of three two-month cycles of cyclophosphamide, methotrexate, vincristine, carboplatin, and etoposide. Intraventricular methotrexate was also administered in 36 doses through an implanted subcutaneous reservoir, beginning 2 to 4 weeks after surgery. The five-year progression-free and overall survival rates $(+/-\mathrm{SE})$ were $82+/-0 \%$ and $93+/-6 \%$ for 17 patients with complete resection, $50+/-13 \%$ and $56+/-14 \%$ for 14 with residual tumor, $33+/-14 \%$ and $38+/-15 \%$ for 12 with macroscopic metastases, and $68+/-8 \%$ and $77+/-8 \%$ in 31 patients without macroscopic metastases. Tumor relapse occurred in 9 of 29 patients without residual disease after chemotherapy. Independent risk factors for tumor relapse were desmoplastic form of medulloblastoma (20 patients), metastatic disease, and age younger than 2 years. Asymptomatic leukoencephalopathy was detected by MRI in 19 of 23 patients. After treatment with chemotherapy, the mean IQ was significantly lower than that of healthy controls of the same age but higher than that of patients treated by radiotherapy in a previous trial. (Rutkowski S, Bode U, Deinlein F et al. Treatment of early childhood medulloblastoma by postoperative chemotherapy alone. N Engl J Med March 10, 2005;352:978-986). (Reprints: Dr Rutkowski, Department of Pediatric Oncology, Children's Hospital, University of Wurzburg, Josef-Schneider Str 2, D-97080 Wurzburg, Germany or at rutkowski@mail.uni-wuerzburg.de). 